Conclusions In this the largest series to date, the accuracy of vascular staging by EUS was found to be impaired by biliary SEMS. We recommend that patients who require biliary drainage and EUS staging should have EUS before stent placement.

\section{P57 LAPAROSCOPIC ASSISTED ENDOSCOPIC MUCOSAL RESECTION OF A COMPLEX CAECAL POLYP}

${ }^{1}$ Jody Parker*, ${ }^{1,2}$ Sunil Dolwanit, ${ }^{2}$ Michael Davies. ${ }^{1}$ Cardiff University, Cardiff, UK; ${ }^{2}$ University Hospital of Wales, Cardiff, UK

10.1136/gutjnl-2020-bsgcampus. 132

Introduction Laparoscopic Assisted Endoscopic Mucosal Resection (Laparoscopic EMR) can offer organ preservation in complex colonic polyps deemed too challenging even with expert therapeutic endoscopy input for colonoscopic resection alone.

Methods This 64 year old male was diagnosed through bowel screening with a $25 \mathrm{~mm}$ laterally spreading polyp in the caecum around the appendix orifice. The patient was assessed by a surgeon, an advanced endoscopist and discussed at a Complex Polyp Multi Disciplinary Team Meeting. Due to the extent of appendix orifice involvement, endoscopic intervention alone was deemed unlikely to be successful and a Laparoscopic EMR was recommended. The patient was placed in the Lloyd David position. For this caecal polyp $10 \mathrm{~mm}$ supraumbilical, $5 \mathrm{~mm}$ suprapubic and $5 \mathrm{~mm}$ left lower quadrant laparoscopic ports were inserted. Lateral mobilisation of the caecum was performed to facilitate laparoscopic assistance during the colonoscopic procedure. The introduction of a tape around the terminal ileum prevented inflation of the small bowel from the colonoscope. Thorough laparoscopic and endoscopic assessment ensured there was no signs of malignant change in the polyp. Piecemeal EMR was performed with laparoscopic assistance achieving complete clearance of lateral margins as visualised through the colonoscopic view. Laparoscopic invagination of the appendix enabled full resection of the polyp from the orifice. Finally the caecum was assessed to exclude evidence of immediate complications.

Results There were no intra or post operative complications. The patient was discharged the day following the procedure and histology confirmed a tubulovillous adenoma with low grade dysplasia. A check colonoscopy will be performed three months after the procedure.

Conclusions Laparoscopic EMR enables resection of complex colonic polyps that are not amenable to expert colonoscopic intervention alone whilst avoiding bowel resection and its associated risks.

\section{P58 LAPAROSCOPIC ASSISTED ENDOSCOPIC MUCOSAL RESECTION REDUCES THE NEED FOR BOWEL RESECTION FOR COMPLEX COLONIC POLYPS}

${ }^{1}$ Jody Parker*, ${ }^{2}$ Michael Davies, ${ }^{1,2}$ Sunil Dolwani. ${ }^{1}$ Cardiff University, Cardiff, UK; ${ }^{2}$ University Hospital of Wales, Cardiff, UK

\subsection{6/gutjnl-2020-bsgcampus. 133}

Introduction The incidence of benign complex colonic polyps is increasing. Lesion size, position or access difficulties may restrict endoscopic removal even with expert therapeutic colonoscopist input. Laparoscopic Assisted Endoscopic Mucosal
Resection (Laparoscopic EMR) may facilitate polyp removal and avoid colonic resection and its risks. Our aim was to assess outcomes after Laparoscopic EMR for the removal of selected complex colonic polyps.

Methods A retrospective review was performed of consecutive Laparoscopic EMR patients between September 2008 and October 2018 in a tertiary referral unit. All included lesions and patients were prospectively assessed and discussed in a Complex Polyp Multi Disciplinary Team Meeting. Decisions confirming the suitability for Laparoscopic EMR were made when complex colonoscopic intervention alone was not feasible due to polyp size or access difficulties or had previously been unsuccessful.

Results There were 50 patients treated in the series. Median polyp size was $40 \mathrm{~mm}$ (range $8-90 \mathrm{~mm}$ ) with $50 \%$ located in the caecum. Indications for laparoscopic EMR included difficult access (46\%), size (28\%) or both (24\%). Endoscopic assisted laparoscopic appendicectomy was required in $8 \%$ of patients. There was a $12 \%$ intraoperative conversion rate to bowel resection. Postoperative complications included one bleed requiring re-operation and two urinary retentions. Median length of stay was 1 day. Final histology demonstrated malignancy in 5 polyps $(9.6 \%)$. Two of these had been converted during their initial procedure as cancer was suspected during the intra operative assessment of the polyp. The remaining three had a subsequent bowel resection after discharge. Incidence of residual disease at first surveillance was $10 \%$ and all were successfully treated by colonoscopy. There was no polyp recurrence during colonoscopic surveillance over a median follow up of 77.5 months.

Conclusions Laparoscopic EMR avoided bowel resection in $82 \%$ of patients selected for this procedure. This technique provides a safe option for complex colonic polyps where advanced colonoscopic intervention alone is either unfeasible or unsuccessful with the benefits of low morbidity and excellent long term outcomes.

\section{P59 ARTIFICIAL INTELLIGENCE INCREASES ADENOMA DETECTION EVEN IN 'HIGH-DETECTOR' COLONOSCOPY: EARLY EVIDENCE FOR HUMAN: MACHINE INTERACTION}

Mehul Patel*, Shraddha Gulati, Sarah O’Neil, Nicholas Wilson, Sophie Williams, Shonette Charles-Nurse, Guy Chung-Faye, Rajaventhan Srirajaskanthan, Amyn Haji, Bu'Hussain Hayee. King's College Hospital NHS Foundation Trust, London, UK

\subsection{6/gutjnl-2020-bsgcampus. 134}

Introduction Artificial intelligence (AI)-driven polyp detection (PD) modules to assist colonoscopy in real time are now commercially available. Early controlled studies suggests that polyp detection rates (and by inference adenoma detection rates $(\mathrm{ADR})$ ) and mean adenomas per patient (MAP) is improved by using such a module. We wished to examine the real-world effect of AI-PD on ADR and MAP.

Methods As service evaluation, the GI Genius ${ }^{\mathrm{TM}}$ module (Medtronic, Ltd) was used by three 'high-detector' colonoscopists for index colonoscopy procedures over three months (the 'active' period). Data was collected prospectively. Results were compared to the three months immediately preceding use (the 'baseline'), as well as for two months after the active period, when the device was intentionally not used ('inactive'). Withdrawal time included time taken to resect lesions.

Results Data from 163 index procedures were analysed (mean age $65.8 \pm 4.8 \mathrm{y} ; 69 \mathrm{~F}$ ). During the active period, ADR increased 